\title{
AUTOEFICÁCIA DE ALUNOS DO ENSINO MÉDIO E NÍVEL DE ESCOLARIDADE DOS PAIS
}

\author{
Daniela Couto Guerreiro-Casanova \\ Doutoranda em Educação - Universidade Estadual de Campinas - Bolsista FAPESP \\ Marilda Aparecida Dantas \\ Doutoranda em Educação - Universidade Estadual de Campinas \\ Roberta Gurgel Azzi \\ Doutora em Educação e docente Universidade Estadual de Campinas
}

\begin{abstract}
Resumo
O objetivo deste estudo foi analisar diferenças entre as percepções de autoeficácia acadêmica e uso de estratégias de estudo e aprendizagem em aulas de língua portuguesa de estudantes do ensino médio de escolas públicas tendo como critério o nível de estudo dos pais. Participaram 534 estudantes do ensino médio de escolas públicas do estado de São Paulo. Foram utilizados três instrumentos de autorrelato: questionário de caracterização, Escala de Autoeficácia Acadêmica e o Inventário de Estratégias de Estudo e Aprendizagem. Os resultados encontrados por meio do teste de Kruskall-Wallis indicaram diferenças significativas para as crenças de autoeficácia acadêmica dos estudantes de acordo com os diferentes níveis de estudo das mães e dos pais. Os resultados do uso de estratégias de estudo e aprendizagem dos estudantes não apresentaram diferenças significativas quando considerados os níveis de estudos das mães e dos pais. Sugerem-se pesquisas sobre envolvimento parental nas atividades escolares de seus filhos.
\end{abstract}

Palavras-chave: motivação, pais, estratégias de estudo.

\section{HIGH SCHOOL STUDENT'S SELF-EFFICACY AND PARENTS SCHOOLING LEVEL}

\begin{abstract}
The aim of this study was to analyze differences between the perceptions of academic self-efficacy and the use of learning and study strategies in Portuguese language classes of high school students from public schools, having as criteria the parents schooling levels. Participants were 534 public high school students in the state of São Paulo. The following instruments were used: High School Students Academic SelfEfficacy Scale and Learning and Study Strategies Inventory, having used its dimension named Information Processing. The results found by the Kruskal-Wallis test indicated significant differences in academic self-efficacy beliefs of students according to the different levels of schooling of mothers and fathers. The results of the use of learning and study strategies showed no significant differences when considering the level of studies of mothers and fathers. Further research is suggested on parental involvement in school activities.
\end{abstract}

Keywords: motivation, parents, study strategies. 


\title{
LA AUTO-EFICACIA DE ESTUDIANTES DE SECUNDARIA E NIVEL EDUCATIVO DE LOS PADRES
}

\begin{abstract}
Resumen
El objetivo de este estudio fue analizar las diferencias entre las percepciones de autoeficacia académica y el uso de estrategias de estudio y el aprendizaje de las clases de portugués para estudiantes de secundaria de escuelas públicas teniendo como criterio el nivel de escolaridad de los padres. 534 estudiantes asistieron a las escuelas secundarias públicas en el estado de São Paulo. Se han utilizado tres instrumentos de auto-informe: cuestionario, caracterización, la auto-eficacia de escala y el Inventario de los estudios académicos y estrategias de aprendizaje. Los resultados de la prueba de Kruskal-Wallis indicaron diferencias significativas en el ámbito académico las creencias de auto-eficacia de los estudiantes de acuerdo a los diferentes niveles de escolaridad de las madres y los padres. Los resultados de la utilización de estrategias de estudio y el aprendizaje de los estudiantes no mostraron diferencias significativas al considerar el nivel de estudios de los padres y madres. Se sugiere estudios sobre la participación de los padres en las actividades escolares de sus hijos.
\end{abstract}

Palabras clave: motivación, padres, estrategias de estudio.

\section{INTRODUÇÃO}

Aspectos socioculturais de pares, família, cultura e comunidade exercem um papel fundamental no desenvolvimento, realização e motivação do estudante. Dentre esses se destaca, neste texto, o papel da família na motivação para aprender dos estudantes. O objetivo deste estudo foi identificar e analisar diferenças entre as percepções de autoeficácia acadêmica e uso de estratégias de estudo e aprendizagem em aulas de língua portuguesa de estudantes do ensino médio de escolas públicas de acordo com o nível de estudo dos pais e das mães.

Segundo Schunk, Pintrich e Meece (2008) o estilo parental exercido em casa tem importantes implicações para os resultados escolares. O envolvimento parental na vida acadêmica dos filhos indica positividade com a motivação para aprender ao refletir uma clara mensagem de que a educação é para ser valorizada. No contexto familiar, Bandura (1997) comenta que as influências dos pais para capacitar os filhos a interagirem efetivamente nos ambientes prediz 0 curso do desenvolvimento cognitivo, sendo que os níveis socioeconômicos pouco contribuem para esse desenvolvimento quando variações na capacidade parental são controladas. Como origem, as primeiras experiências de eficácia dos estudantes são centradas na família, com o passar do tempo, os pares assumem fortemente este papel. Mesmo dentro de uma mesma família, as experiências se diferenciam em função da estrutura familiar quanto a tamanho, distribuição de gênero e ordem de nascimento quanto a filhos. Bandura (1997, p.3) define a 
crença de autoeficácia como a "crença de alguém em sua capacidade em organizar e executar cursos de ações requeridos para produzir certas realizações". Com o desenvolvimento cognitivo por meio de experiências exploratórias, modelação e instruções, os filhos gradativamente desenvolvem a autoavaliação de suas habilidades. O autoconhecimento gerado a partir da avaliação da capacidade das habilidades serve para julgar a eficácia como guia a ações em determinadas situações. Bandura (2006) assinala que para cada período do desenvolvimento humano é necessário uma nova competência, frente às mudanças e oportunidades de crescimento pessoal. A adolescência representa uma dessas fases, pois eles precisam gerenciar transições de papéis do ponto de vista biológico, social e educacional.

Em relação aos aspectos educacionais, filhos de famílias com melhores recursos educacionais podem vivenciar ambientes que proporcionem experiências mais enriquecedoras para estimular a crença de autoeficácia acadêmica (Schunk \& Meece, 2006). Esta crença tem sido apontada como preditora de ações desejáveis para promover a aprendizagem escolar. A autoeficácia acadêmica é definida como as crenças de um estudante em sua capacidade em organizar e executar cursos de ações requeridos para produzir certas realizações, referentes aos aspectos intelectuais e de aprendizagem, coloca-se como um constructo necessário à postura requerida do estudante (Bandura, 1993).

Estudos têm demonstrado (Bandura, 1993; 1995; Zimmerman, 1995; Zimmerman \& Cleary, 2006) que estudantes com alta percepção de eficácia acadêmica demonstram-se mais engajados cognitivamente durante as aulas, o que os auxilia a desenvolver competências educacionais, como também mais persistentes frente aos desafios escolares, além de demonstrarem maior disponibilidade para tarefas relativas aos estudos mesmo quando há outras atividades sociais para se fazer. Apesar de não garantir a habilidade, a crença de autoeficácia atua como mediadora da motivação para aprender (Azzi \& Polydoro, 2010), auxiliando a seleção e a utilização de diversas estratégias de aprendizagem, de modo a possibilitar que o estudante busque encontrar as estratégias mais apropriadas para cada tipo de atividade a ser desenvolvida (Bandura, 1993; 1995; Zimmerman, 1995).

A adoção de estratégias de aprendizagem distintas para processar as informações se faz mais necessária devido ao advento tecnológico que acarretou 
uma enxurrada de informações provindas de novos sistemas de produção, comunicação e conservação da informação. Isso tornou a aprendizagem mais diversa e complexa, multiplicando as possibilidades de aprender diversos conteúdos e em diversas condições. As estratégias de estudo e aprendizagem são definidas como um conjunto de estratagemas destinado a gestão pessoal para o processo de aprendizagem. As estratégias direcionadas ao processamento da informação são entendidas como seleção, organização, elaboração e repetição da informação, associadas à área cognitiva da autorregulação da aprendizagem, são meios que os estudantes utilizam para manejar os dados. Outras estratégias são relevantes do processo de aprendizagem, tais como o planejamento, a supervisão, a revisão e valorização, estratégias afetivas e motivacionais, gestão de recursos como tempo e ajuda (Valle et al., 2008).

O uso de estratégias de estudo e aprendizagem tem sido investigado por meio de programas de intervenção, como o trabalho de Rosário et al. (2007). Esses autores aplicaram um programa relacionado à aprendizagem autorregulada em estudantes portugueses iniciantes do ensino superior, que entre outros conteúdos, abordou o ensino de estratégias. Por se tratar de uma pesquisa quase-experimental, os autores realizaram pré e pósteste, tendo a aplicação do programa neste intervalo. Os resultados indicaram que os alunos do grupo experimental demonstraram um maior conhecimento de estratégias de aprendizagem como também melhor qualidade de realização nas tarefas solicitadas ao final da aplicação do programa, o que não ocorreu com o grupo controle na análise do pré e pósteste.

Segundo o modelo teórico de Greene, Miller, Crowson, Duke e Akey (2004) a autoeficácia acadêmica prediz o uso de estratégias de estudo e aprendizagem, contribuindo para o desempenho dos estudantes, dentre outras variáveis. 0 desempenho acadêmico também é considerado um dos melhores preditores de aspirações profissionais e, conseqüentes, decisões de carreira (Brown \& Lent, 2006). No entanto, além do desempenho acadêmico, Lopes (2010) verificou que o grau de envolvimento dos pais quanto à vida escolar e expectativas para o futuro de seus filhos relacionam-se positivamente com o nível de aspiração dos jovens.

Inserido no contexto de investigações que exploram a autoeficácia acadêmica, este estudo busca responder se a percepção de Autoeficácia Acadêmica e de uso de Estratégias de Estudo e de Aprendizagem apresentam 
diferenças de acordo com o nível de estudo dos pais e das mães? A presente pesquisa destina-se a investigar tal problematização, especificamente em relação às aulas de língua portuguesa de estudantes do ensino médio.

\section{MÉTODO}

A presente pesquisa caracteriza-se como quantitativa e descritiva, sendo parte de um estudo maior (Zambom, Azzi, Polydoro, De Rose \& GuerreiroCasanova, 2011) que buscou investigar variáveis motivacionais em estudantes do ensino médio. O recorte pautado na teoria social cognitiva, aqui realizado, refere-se aos constructos de Autoeficácia Acadêmica e Estratégias de Estudo e Aprendizagem.

\section{Procedimento}

O projeto de pesquisa foi submetido ao Comitê de Ética em Pesquisa de uma instituição de ensino superior e aprovado pelo parecer no 1185/2009. Após a aprovação pelo CEP a coleta de dados foi planejada, tendo início pelo contato com as diretorias de ensino para a respectiva aprovação. Em seguida foi realizado contato com os gestores das instituições de ensino a fim de programar o agendamento da coleta de dados. A participação das escolas foi assegurada após a autorização da diretora de cada unidade escolar, via assinatura da Carta de Autorização.

A coleta foi realizada na aula de língua portuguesa e os estudantes foram orientados a responderem os instrumentos considerando o contexto dessa disciplina. Todos os participantes menores de idade foram autorizados pelos seus pais e/ou responsáveis a integrarem a amostra da pesquisa, por meio da assinatura do Termo de Consentimento Livre e Esclarecido. Para os participantes maiores de 18 anos foi solicitado apenas o próprio consentimento via assinatura do Termo de Consentimento Livre e Esclarecido. A coleta de dados foi realizada em sala de aula, com a presença do pesquisador para as devidas orientações de preenchimento, numa única sessão com duração média de 30 minutos.

\section{Participantes}

Esse estudo foi realizado em nove escolas de ensino médio localizadas no estado de São Paulo. A fim de se obter diversidade da amostra foram convidadas a participar da pesquisa escolas localizadas em diferentes regiões do estado, de 
modo a contemplar regiões metropolitanas e cidades menores, localizadas no interior do estado.

Foram participantes desta pesquisa 534 estudantes do ensino médio, com 64,6\% ( $n=345)$ do sexo feminino. Em relação à idade, a amostra obteve estudantes entre 14 e 24 anos, com idade média de 15,97. Da amostra geral, 183 estudantes cursavam a $1^{\text {a }}$ série, 193 estudantes cursavam a $2^{a}$ série e 155 estudantes cursavam a $3^{a}$ série do ensino médio e 3 participantes não responderam essa questão. Desta amostra, 50,93\% $(n=272)$ dos estudantes frequentavam a escola no turno matutino, $41,01 \%(n=219)$ no turno noturno e 7,86\% ( $n=42)$ no turno vespertino, sendo que 1 participante não respondeu essa questão. Praticamente, a totalidade dos estudantes pesquisados pretende concluir o ensino médio $(99,6 \%, n=529)$ e ingressar no ensino superior $(93,4 \%$, $\mathrm{n}=499)$. Em relação à condição de exercer atividade de trabalho remunerado, 30,8\% ( $n=163)$ dos estudantes declararam-se como trabalhadores. Na Tabela 1, pode-se observar a distribuição do nível de estudos dos pais e das mães desses 534 estudantes participantes.

Tabela 1.

Número de estudantes que declaram o nível de estudo de seus pais.

\begin{tabular}{ccccc}
\hline Nível de ensino & \multicolumn{2}{c}{ Estudo do pai } & \multicolumn{2}{c}{ Estudo da mãe } \\
& $\mathrm{N}$ & $\mathrm{F}$ & $\mathrm{N}$ & $\mathrm{F}$ \\
\hline Ensino Fundamental Incompleto (EFI) & 176 & $33 \%$ & 171 & $32 \%$ \\
Ensino Médio Incompleto (EMI) & 55 & $10,3 \%$ & 73 & $13,7 \%$ \\
Ensino Superior Incompleto (ESI) & 17 & $3,2 \%$ & 18 & $3,4 \%$ \\
Ensino Fundamental Completo (EFC) & 40 & $7,5 \%$ & 60 & $11,2 \%$ \\
Ensino Médio Completo (EMC) & 109 & $20,4 \%$ & 119 & $22,3 \%$ \\
Ensino Superior Completo (ESC) & 34 & $6,4 \%$ & 24 & $4,5 \%$ \\
Não Sabe (NS) & 99 & $18,5 \%$ & 69 & $12,9 \%$ \\
Não Responderam & 4 & $0,7 \%$ & - & - \\
\hline Total & 534 & $100 \%$ & 534 & $100 \%$ \\
\hline
\end{tabular}

Ao observar a Tabela 1, pode-se perceber uma concentração de estudantes com pais $(33 \%)$ e mães (32\%) que cursaram até o ensino fundamental incompleto. Seguindo-se estudantes com pais $(20,4 \%)$ e mães $(22,3 \%)$ que cursaram o ensino médio completo. Outro dado verificado foi o agrupamento de estudantes que declararam não saber o nível de ensino de seus pais $(18,5 \%)$ e/ou mães (12,9\%). 


\section{Materiais}

Para a realização desta pesquisa foram utilizados três instrumentos de coleta de dados, os quais serão apresentados a seguir:

\section{Questionário de caracterização}

Este instrumento foi elaborado pelas pesquisadoras, com objetivo de obter informações sobre as características pessoais dos estudantes que participaram da pesquisa. As características foram idade, sexo, série, turno, condição de trabalho e nível de estudo dos pais e das mães.

\section{Escala de Autoeficácia Acadêmica de Ensino Médio}

Esta escala foi desenvolvida a partir da escala de Autoeficácia na Formação Superior (Polydoro \& Guerreiro-Casanova, 2010), passando por adaptações de linguagem e de contexto necessárias para a compreensão dos estudantes do ensino médio. Após essa adaptação inicial, foi realizado o processo de reflexão falada com 10 estudantes de ensino médio, aos quais foram solicitadas as opiniões sobre a compreensão dos itens que integravam a escala e sobre a aplicabilidade desses itens ao contexto educacional do ensino médio. Tal procedimento desencadeou pequenos ajustes de redação nos itens da escala. Após a realização da avaliação das propriedades psicométricas, a escala passou a ser composta por 16 itens. Para respondê-los, os estudantes foram orientados a pensarem sobre a experiência escolar atual. A partir disso, os alunos deveriam indicar o quanto eles se sentiam capazes de realizar as situações propostas em cada um dos itens, considerando um contínuo entre 1, para pouco capaz, e 7 para muito capaz. A Escala demonstrou consistência interna de 0,886. Os itens organizaram-se em três dimensões, conforme pode ser observado na Figura 1.

\section{Inventário de Estratégias de Estudo e Aprendizagem}

Este instrumento foi originalmente elaborado por Weinstein e Palmer (1990) e, posteriormente, traduzido para o português de Portugal por Figueira (1994). No Brasil, foi traduzido e validado por Bartalo (2006). Nesta pesquisa foi utilizada apenas a dimensão Processamento de Informação, a qual pode ser observada na Figura 2, composta por 12 dos 88 itens que compõem o instrumento em questão. Essa dimensão apresenta consistência interna de a=0,82. 


\begin{tabular}{|c|c|c|c|}
\hline Dimensões & Destina-se a verificar & $\begin{array}{l}\text { Número } \\
\text { de itens }\end{array}$ & Exemplo de item \\
\hline $\begin{array}{c}\text { Autoeficácia } \\
\text { para } \\
\text { Aprender }\end{array}$ & $\begin{array}{l}\text { Confiança percebida na } \\
\text { capacidade de mobilizar esforços } \\
\text { para se envolver nas atividades } \\
\text { escolares considerando os } \\
\text { aspectos cognitivos, } \\
\text { motivacionais e } \\
\text { comportamentais pertinentes ao } \\
\text { processo de aprender e ao } \\
\text { desempenho acadêmico. }\end{array}$ & 8 itens & $\begin{array}{c}\text { Quanto eu sou capaz } \\
\text { de motivar-me para } \\
\text { fazer as } \\
\text { atividades/tarefas } \\
\text { relacionadas a esta } \\
\text { disciplina? }\end{array}$ \\
\hline $\begin{array}{l}\text { Autoeficácia } \\
\text { para atuar } \\
\text { na vida } \\
\text { escolar }\end{array}$ & $\begin{array}{l}\text { Confiança percebida na } \\
\text { capacidade de atuar na vida } \\
\text { escolar, considerando o } \\
\text { envolvimento colaborativo com } \\
\text { os pares e a escola/instituição. }\end{array}$ & 4 itens & $\begin{array}{l}\text { Quanto eu sou capaz } \\
\text { de contribuir com } \\
\text { ideias para a melhoria } \\
\text { da minha escola? }\end{array}$ \\
\hline $\begin{array}{l}\text { Autoeficácia } \\
\text { para } \\
\text { decisão de } \\
\text { carreira }\end{array}$ & $\begin{array}{c}\text { Confiança percebida na } \\
\text { capacidade de buscar } \\
\text { informações e planejar ações } \\
\text { relevantes para a decisão de } \\
\text { carreira. }\end{array}$ & 4 itens & $\begin{array}{l}\text { Quanto eu sou capaz } \\
\text { de definir com } \\
\text { segurança o que } \\
\text { pretendo seguir como } \\
\text { profissão dentre as } \\
\text { diversas possibilidades } \\
\text { de atuação profissional } \\
\text { que existem? }\end{array}$ \\
\hline
\end{tabular}

Figura 1.

Descrição das dimensões do instrumento de Autoeficácia Acadêmica.

Para responder a dimensão do Inventário de Estratégias de Estudo e Aprendizagem, o estudante foi orientado a pensar sobre seu comportamento habitual relativo às estratégias citadas no instrumento. Ao responder, o estudante pôde escolher entre um contínuo de 1 a 5 , de modo que 1 representava um comportamento nunca realizado pelo estudante e 5 representava um comportamento sempre realizado pelo estudante durante suas atividades de estudo e aprendizagem.

\section{RESULTADOS}

As análises estatísticas foram realizadas por meio do programa SPSS (versão 17). Os instrumentos que apresentaram menos de $90 \%$ de respostas foram excluídos. A confiabilidade da digitação foi conferida aleatoriamente considerando-se $20 \%$ dos dados. 


\begin{tabular}{|c|c|c|}
\hline Dimensão & Destina-se a verificar & Exemplo de item \\
\hline $\begin{array}{c}\text { Processamento } \\
\text { da Informação }\end{array}$ & $\begin{array}{c}\text { Identifica a utilização de } \\
\text { estratégias verbais e simbólicas, } \\
\text { o monitoramento da } \\
\text { compreensão e do raciocínio, } \\
\text { bem como a criação de pontes } \\
\text { entre o que já sabem e o que } \\
\text { estão tentando aprender e se } \\
\text { lembrar. }\end{array}$ & $\begin{array}{c}\text { Tento identificar as idéias } \\
\text { professor desta disciplina } \\
\text { está dando aula. }\end{array}$ \\
\hline
\end{tabular}

Figura 2.

Descrição da dimensão Processamento da Informação do Inventário de Estratégias de Estudo e Aprendizagem.

Para iniciar a análise utilizou-se o teste Kolmogorov-Sminov e observou-se que os dados eram não-paramétricos. Em função disso, decidiu-se utilizar o teste de Kruskal Wallis a fim de verificar diferenças entre os grupos, uma vez que foi identificado mais de 2 grupos independentes. Os grupos foram organizados considerando os níveis de estudos dos pais e das mães, de modo a identificar as diferenças entre o constructo Autoeficácia Acadêmica (escala total) e suas dimensões Autoeficácia para Aprender, Autoeficácia para Atuar na Vida Escolar e Autoeficácia para Decisão de Carreira, como também o constructo de Estratégias de Estudo e Aprendizagem. Os resultados serão apresentados em duas partes, inicialmente a exploração dos dados relacionados às crenças de Autoeficácia Acadêmica e às Estratégias de Estudo e Aprendizagem correspondentes ao nível de estudo das mães, que podem ser observados na Tabela 2, seguindo-se dos resultados correspondentes ao nível de estudo dos pais, que podem ser verificados na Tabela 3.

Ao considerar a Autoeficácia Acadêmica segundo o nível de estudo da mãe $\left(x^{2}=22,273\right)$ verifica-se diferença significativa $(p=0,001)$ para o total da escala, observado na Tabela 2 . Os estudantes filhos de mãe que cursaram o ensino superior completo apresentaram maior percepção de Autoeficácia Acadêmica, ficando-se com o primeiro posto médio (média rank=251,55). A seguir, com o segundo posto médio (média rank $=248,08$ ) encontram-se os estudantes cujas mães cursaram o ensino fundamental incompleto. O terceiro posto (média rank $=247,57$ ) foi obtido pelos estudantes filhos de mães que cursaram o ensino fundamental completo. No quarto posto (média rank $=238,69$ ) encontram-se os 
estudantes cujas mães cursaram o ensino superior de modo incompleto. O quinto posto médio (média rank $=232,04$ ) foi obtido pelos estudantes filhos de mãe que cursaram o ensino médio completo, seguindo-se, em sexto posto médio (média rank $=198,74)$, dos estudantes filhos de mães com o ensino médio incompleto. Com menor percepção de Autoeficácia Acadêmica, em sétimo posto médio (média rank=163,89), ficaram os estudantes que não sabiam o nível de estudo de suas mães.

Tabela 2.

Resultados de Autoeficácia Acadêmica e de Estratégias de Estudo e Aprendizagem segundo o nível de estudo das mães.

\begin{tabular}{|c|c|c|c|c|c|c|c|c|c|c|}
\hline \multirow[t]{2}{*}{$\begin{array}{l}\text { Posto } \\
\text { médio }\end{array}$} & \multicolumn{2}{|c|}{$\begin{array}{c}\text { Autoeficácia } \\
\text { Acadêmica }\end{array}$} & \multicolumn{2}{|c|}{$\begin{array}{l}\text { AE para } \\
\text { Aprender }\end{array}$} & \multicolumn{2}{|c|}{$\begin{array}{c}\text { AE para } \\
\text { Atuar na } \\
\text { Vida Escolar }\end{array}$} & \multicolumn{2}{|c|}{$\begin{array}{c}\text { AE para } \\
\text { Decisão de } \\
\text { Carreira }\end{array}$} & \multicolumn{2}{|c|}{$\begin{array}{l}\text { Estratégias de } \\
\text { Estudo e } \\
\text { Aprendizagem }\end{array}$} \\
\hline & NE & $M R$ & $N$ & $M R$ & $\mathrm{NE}$ & $M R$ & $\mathrm{NE}$ & $M R$ & $\mathrm{NE}$ & $M R$ \\
\hline 1 & & $-1-1$ & & & C & 5 & $\mathrm{C}$ & & 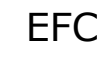 & 26 \\
\hline 20 & 11 & $24 \varepsilon$ & & 5 & EFI & D, & SI & & EFI & 253,68 \\
\hline 30 & $=\mathrm{C}$ & $y$ & ESI & & EMC & 261 & EFC & 27 & EMI & 237,22 \\
\hline 40 & ST & 238,69 & EMC & 2 & SI & 258 & $\mathrm{FI}$ & 2 & ESI & 232,68 \\
\hline $5^{\circ}$ & $\mathrm{Mr}$ & 232,04 & FMT & 6 & $\mathrm{FC}$ & 236 & MC & 5 & NS & 218,52 \\
\hline $6 c$ & & 198,74 & & 7 & II & 228, & $1 \mathrm{I}$ & -5 & EMC & 213,47 \\
\hline 70 & IVS & 103,0 & 10 & 192,63 & NS & 197,21 & NS & 226,73 & ESC & 181,52 \\
\hline & \multicolumn{2}{|c|}{$x^{2}=22,273$} & \multicolumn{2}{|c|}{$x^{2}=21,413$} & \multicolumn{2}{|c|}{$x^{2}=16,734$} & \multicolumn{2}{|c|}{$x^{2}=9,482$} & \multicolumn{2}{|c|}{$x^{2}=11,021$} \\
\hline & \multicolumn{2}{|c|}{$\mathrm{p}=0,001 *$} & \multicolumn{2}{|c|}{$=0,002 *$} & \multicolumn{2}{|c|}{$\mathrm{p}=0,010^{*}$} & \multicolumn{2}{|c|}{$\mathrm{p}=0,148^{* *}$} & \multicolumn{2}{|c|}{$\mathrm{p}=0,88^{* *}$} \\
\hline
\end{tabular}

*Diferença significativa / **Diferença não significativa / NE=Nível de Estudo / MR=Média Rank

Para a dimensão Autoeficácia para Aprender também foi encontrada diferença significativa $(p=0,002)$ de acordo com o nível de estudo das mães $\left(x^{2}=21,413\right)$. A maior percepção (média rank=287,61) de Autoeficácia para Aprender foi demonstrada pelos estudantes filhos de mães que estudaram apenas o ensino fundamental. O segundo posto médio (média rank=269,05) foi obtido pelos estudantes cujas mães estudaram o ensino fundamental de modo incompleto. Com o terceiro posto (média rank=257,76) ficaram os estudantes filhos de mães que estudaram o ensino superior incompleto. No quarto posto (média rank $=236,42$ ) pode ser observado os estudantes filhos de mães que cursaram o ensino médio completo, seguindo-se dos estudantes filhos de mães que cursaram o ensino médio incompleto com o quinto posto médio (média rank $=228,76)$. Em sexto, estão os estudantes cujas mães cursaram o ensino 
superior completo (média rank=205,94). Com o último posto médio, ou seja, demonstrando menor percepção de Autoeficácia para Aprender, ficaram os estudantes que não sabiam qual o nível de estudo de suas mães (média rank=192,63).

Também foram encontradas diferenças significativas $(p=0,010)$ para a percepção de autoeficácia quando considerada a dimensão Autoeficácia para Atuar na Vida Escolar $\left(X^{2}=16,734\right)$. A maior percepção de Autoeficácia para Atuar na Vida Escolar foi demonstrada pelos estudantes cujas mães cursaram o ensino superior (média rank=306,55). O segundo posto médio (média rank=266,73) foi obtido pelos estudantes filhos de mães que cursaram o ensino fundamental incompleto. O terceiro posto médio (média rank=261,35) ficou com os estudantes cujas mães cursaram o ensino médio. Os filhos de mães que cursaram o ensino superior de modo incompleto obtiveram o quarto posto médio (média rank=258,29). O quinto posto médio (média rank=236,39) foi obtido pelos estudantes filhos de mães que cursaram o ensino fundamental completo. Com o sexto posto médio (média rank=228,41) ficaram os filhos de mães que estudaram até o ensino médio, mas de modo incompleto. $E$, a percepção mais baixa de Autoeficácia para Atuar na Vida Escolar (média rank=197,21) foi demonstrada pelos estudantes que não sabiam até que nível suas mães tinham estudado.

Ao se considerar a dimensão Autoeficácia para Decisão de Carreira não foram identificadas diferenças significativas $(p=0,148)$ de acordo com o nível de estudo das mães dos estudantes aqui pesquisados $\left(X^{2}=9,482\right)$. Na Tabela 2 , pode ser observado as médias ranks demonstradas de acordo com o nível de estudo das mães, as quais não serão aqui comentadas devido a falta de diferença significativa.

Em relação ao uso das Estratégias de Estudo e de Aprendizagem também não foram verificadas diferenças significativas $(p=0,88)$ de acordo com o nível de estudo das mães $\left(X^{2}=11,021\right)$. Na Tabela 2 , pode-se observar a ordem em que o uso de Estratégias de Estudo e de Aprendizagem se organizaram segundo o nível de estudo das mães, mas essas não serão comentadas pela falta de diferença significativa.

Sobre os resultados quanto à escolaridade dos pais foram encontradas diferenças significativas também na escala de Autoeficácia Acadêmica, exceto na dimensão de Autoeficácia para Decisão de Carreira. Não foi observada diferença 
significativa também quanto ao uso de Estratégias de Estudo e Aprendizagem pelos estudantes quando comparados aos diferentes níveis de estudo dos pais, conforme Tabela 3.

Considerando os dados da escala de Autoeficácia Acadêmica em seu total $\left(x^{2}=17,894\right)$ notou-se diferenças significativas $(p=0,007)$ em relação aos níveis de estudos dos pais. Em uma análise das médias ranks dos diferentes níveis de estudo dos pais informados pelos estudantes, notou-se que os participantes cujos pais tinham nível superior completo apresentaram maior percepção de Autoeficácia Acadêmica (média rank=268,48), obtendo o primeiro posto, seguido por pais com nível superior incompleto (média rank=245,10), ensino médio incompleto (média rank=236,33), ensino fundamental incompleto (média rank $=235,78$ ), ensino médio completo (média rank $=233,70$ ), ensino fundamental completo (média rank=218,76), e por último, no sétimo posto, aqueles participantes que informaram não saber o nível de estudos dos pais (média rank=175,21).

Tabela 3.

Resultados das diferenças de Autoeficácia Acadêmica e de Estratégias de Estudo e Aprendizagem segundo o nível de estudo dos pais.

\begin{tabular}{|c|c|c|c|c|c|c|c|c|c|c|}
\hline \multirow[t]{2}{*}{$\begin{array}{l}\text { Posto } \\
\text { médio }\end{array}$} & \multicolumn{2}{|c|}{$\begin{array}{l}\text { Autoeficácia } \\
\text { Acadêmica }\end{array}$} & \multicolumn{2}{|c|}{$\begin{array}{l}\text { AE para } \\
\text { Aprender }\end{array}$} & \multicolumn{2}{|c|}{$\begin{array}{c}\text { AE para } \\
\text { Atuar na } \\
\text { Vida Escolar }\end{array}$} & \multicolumn{2}{|c|}{$\begin{array}{c}\text { AE para } \\
\text { Decisão de } \\
\text { Carreira }\end{array}$} & \multicolumn{2}{|c|}{$\begin{array}{l}\text { Estratégias de } \\
\text { Estudo e } \\
\text { Aprendizagem }\end{array}$} \\
\hline & $\mathrm{NE}$ & M R & & & $\mathrm{NE}$ & $M R$ & $\mathrm{NE}$ & M R & N E & $M R$ \\
\hline+ & ESC & 268 & & & SC & 288 & ESC & & EFI & 255,62 \\
\hline 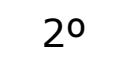 & & & & & ESI & & C & & ESC & 253,48 \\
\hline 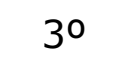 & & & & & 1 & & FI & & EMC & \\
\hline 40 & & & & & MI & & $\mathrm{FC}$ & & EMI & 231,57 \\
\hline 5 & T & & & & MC & 2 & EMI & & ESI & 230,13 \\
\hline 6 & - & & & & EFC & 2 & ESI & & EFC & 205,49 \\
\hline 70 & & 175,21 & IV & 195,23 & NS & 207,87 & NS & 239,69 & NS & 205,00 \\
\hline & \multicolumn{2}{|c|}{$x^{2}=17,894$} & \multicolumn{2}{|c|}{$x^{2=} 15,065$} & \multicolumn{2}{|c|}{$x^{2}=15,024$} & \multicolumn{2}{|c|}{$x^{2}=4,946$} & \multicolumn{2}{|c|}{$x^{2=9,962}$} \\
\hline
\end{tabular}

*Diferença significativa / **Diferença não significativa / NE=Nível de Estudo / $\mathrm{MR}=$ Média Rank

$\mathrm{Na}$ dimensão de Autoeficácia Acadêmica para Aprender $\left(X^{2}=15,065\right)$ as diferenças foram significativas $(p=0,020)$, indicando que o posto médio na percepção deste construto pelos estudantes foram inicialmente com maiores resultados para aqueles cujos pais cursaram estudo superior completo (média 
rank=278,33), depois ensino fundamental incompleto (média rank=258,07), seguido por ensino médio incompleto (média rank=256,95), ensino superior incompleto (média rank=254,25), ensino médio completo (média rank=243,95), ensino fundamental completo (média rank $=236,29$ ) e, com o último posto, os estudantes que não sabiam o nível de estudos dos pais (média rank=195,23).

A dimensão de Autoeficácia para Atuar na Vida Escolar $\left(x^{2}=15,024\right)$ apresentou diferenças significativas $(p=0,020)$. Considerando o posto médio de acordo com nível de estudo dos pais, é possível indicar que aqueles com ensino superior completo apresentaram maior média rank $(288,97)$, seguido por ensino superior incompleto (média rank=282,88), ensino fundamental incompleto (média rank $=262,80$ ), ensino médio incompleto (média rank=257,03), ensino médio completo (média rank $=249,69$ ), ensino fundamental completo (média rank $=213,69$ ) e por fim quando os estudantes não sabiam sobre os estudos dos pais (média rank=207,87).

\section{DISCUSSÃO}

Nesta discussão, primeiro serão analisados os resultados que demonstraram diferenças significativas sobre as percepções de Autoeficácia Acadêmica relativas à disciplina de Língua Portuguesa de acordo com o nível de estudo das mães e pais. Posteriormente serão discutidos os resultados sem diferenças significativas, referentes à dimensão Autoeficácia para Decisão de Carreira e uso de Estratégias de Estudo e Aprendizagem.

Os resultados verificados neste estudo parecem demonstrar que a percepção de Autoeficácia Acadêmica recebe algum tipo de interferência em decorrência do nível dos estudos das mães e dos pais. Pode-se perceber que a maior parte dos primeiros postos (Autoeficácia Acadêmica e dimensão Autoeficácia para Atuar na Vida Escolar) foi obtida por estudantes cujas mães e pais cursaram o ensino superior completo. Esses estudantes parecem perceber as suas crenças levemente mais elevadas, quando comparadas aos demais, tanto na escala total, quanto nas dimensões de Autoeficácia para Aprender (exceto mães) e para Atuar na Vida Escolar. Esses resultados foram observados pelas médias ranks da escala de Autoeficácia Acadêmica, na qual se encontraram diferenças significativas, o que sugere haver alguma interferência de mães e pais mais escolarizados na percepção de crenças de Autoeficácia Acadêmica dos filhos estudantes, como comentado por Bandura (1997) e Schunk e Meece (2006). 
A maior parte dos segundos postos (Autoeficácia Acadêmica, dimensão Autoeficácia para Aprender e dimensão Autoeficácia para Atuar na Vida Escolar) foi obtida por filhos de mães que cursaram o ensino fundamental incompleto. Por que será que estudantes filhos de mães com escolaridade menor apresentam a segunda maior percepção de Autoeficácia Acadêmica? Acredita-se que os pais procuram incentivar os filhos sempre. Como um possível exemplo dessa situação, pode ser citado um trecho de uma reportagem, na qual um casal que estudou até a quinta série diz: "Incentivamos as crianças a estudar para que tenham um futuro melhor que o nosso" (Ogliari \& Rodrigues, 2011, p. A23). Pela concepção teórica, pode-se dizer que o fato desses estudantes terem avançado mais nos níveis de estudo do que suas mães e pais pode ter contribuído para esse resultado positivo; que as experiências de êxito nas tarefas relativas ao aprender serviram de fonte de autoeficácia para esses estudantes.

Quando se observaram os terceiros, os quartos, os quintos e os sextos postos médios tanto em relação às mães quanto aos pais verificou-se falta de padrão das diferenças entre as percepções de Autoeficácia Acadêmica e suas dimensões. Nesses postos, tanto para as mães, quanto para os pais, foi possível verificar que muitas vezes estudantes cujos pais tinham menor nível educacional obtiveram maior posto médio, indicando maior percepção de Autoeficácia Acadêmica.

$\mathrm{Na}$ pesquisa de Rosário et al. (2005) com estudantes portugueses, foram identificados que estudantes com mães e pais com níveis de estudo mais elevados (pós-graduação), seus filhos apresentaram comportamentos frente às tarefas escolares mais favoráveis, atitudes mais positivas e resultados mais proficientes. A discussão desses pesquisadores gira em torno de que pais com maior nível escolar tenderiam a se envolver mais nas atividades escolares dos filhos, a medida que o nível escolar dos pais diminuía, o envolvimento nas atividades escolares dos filhos também decaia. Nos resultados aqui encontrados, quando comparados ao estudo português citado, notou-se que a lógica quanto ao nível de estudo dos pais não permaneceu. Como exemplo da análise dos resultados dos estudantes desta pesquisa, notou-se que apenas os primeiros postos médios apresentaram um padrão (todos tiveram pais com ensino superior completo). Nos postos seguintes tal padrão não se manteve, diferenciando-se do observado com os estudantes portugueses, essa ocorrência pode talvez ser entendida por diferenças culturais entre os países. Embora Schunk, Pintrich e 
Meece (2008) afirmem que a família relaciona-se com a motivação para aprender dos estudantes, de acordo com os dados aqui encontrados, a característica de nível escolar dos pais parece ser sobreposta por outras condições vivenciadas pelos estudantes. Como indicou Valle (2009) nem todos os estudantes precisam seguir a mesma trajetória motivacional.

Duas tendências foram observadas nos resultados. A primeira refere-se aos estudantes que não sabiam o nível de estudo das mães e dos pais. Estes apresentaram em todas as dimensões de Autoeficácia e uso de Estratégias de Estudo e Aprendizagem como a menor média rank. O fato dos estudantes não saberem o nível de estudo de seu pai e/ou de sua mãe causou relativa estranheza nos pesquisadores considerando a quantidade desses sujeitos. Esse dado parece revelar que temas acadêmicos e escolares não são freqüentes na vida familiar desses participantes. A segunda tendência foi de não haver diferenças significativas na dimensão de Autoeficácia Acadêmica para Decisão de Carreira tanto no nível de estudo das mães e dos pais. Isso pode indicar baixas interferências dos pais, em função de sua escolaridade, nesta dimensão, confirmado pelo fato de que a maior parte dos estudantes $(93,4 \%, n=499)$ informou a intenção de cursar o ensino superior.

Segundo Bandura (1997) as crenças de autoeficácia acadêmica são consideradas como uma das fontes preditoras do comportamento. Azzi, Guerreiro-Casanova, Dantas e Maciel (2011) encontraram correlações positivas e significativas entre autoeficácia acadêmica e uso de estratégias de estudo e aprendizagem com estudantes do ensino médio. Nos resultados encontrados na presente pesquisa foi verificada ausência de diferença significativa entre Estratégia de Estudo e de Aprendizagem e o nível de estudo das mães e dos pais. Isso parece indicar que a mãe e o pai não interferem nessa habilidade, de modo significativo. O resultado desta pesquisa parece sugerir que o uso de Estratégias de Estudo e de Aprendizagem necessita ser ensinado, estimulado e/ou desenvolvido na escola, por meio de ações pedagógicas. Sobre estratégias de aprendizagem Almeida (2002) assinalou que o importante é descobrir, junto com o aluno, e ensaiar formas e construção de contextos de facilitação de aprendizagem, nunca como receitas padronizadas. A idéia é proporcionar a diversidade de estratégias, que inclui a apreciação de quando e como pode ser usado, identificar a utilidade em função da eficácia dos resultados em diferentes situações. Focalizar no aprender e no pensar, construção realizada pelo aluno, de 
modo que favoreça a autonomia e a escolha da estratégia em função de suas características pessoais e exigências das situações.

De modo geral, por meio deste estudo foi possível verificar que os níveis dos estudos das mães e dos pais dos estudantes de ensino médio relacionam-se com as crenças de Autoeficácia Acadêmica. Essas relações não são simetricamente proporcionais ao nível dos estudos, ou seja, o fato do pai ou da mãe ter obtido formação em nível superior (o mais alto nível aqui investigado) não garante percepção de Autoeficácia Acadêmica mais alta. Isso parece indicar que outras variáveis aqui não investigadas podem contribuir para a percepção de Autoeficácia Acadêmica dos estudantes do ensino médio.

A maioria dos primeiros postos médios em todas as dimensões estudadas foi obtida por estudantes filhos de pais e mães que cursaram o ensino superior completo, seguindo-se de estudantes cujas mães cursaram o ensino fundamental completo. No entanto, os segundos postos médios foram obtidos, em muitos casos, por estudantes filhos de mães com apenas o ensino fundamental incompleto. Isso parece demonstrar que a relação que os pais e mães cultivam com seus filhos e a vida escolar é mais importante do que o nível do estudo dos pais e ou mães.

Como principais resultados destacam-se que: (1) o nível de estudo das mães e dos pais não exerceram influência de modo padronizado nas percepções de Autoeficácia Acadêmica. Isso parece indicar que um estudante cujo pai não terminou 0 ensino fundamental pode perceber-se como autoeficaz academicamente; e (2) o nível de estudo das mães e dos pais não demonstraram diferenças entre 0 uso de Estratégias de Estudo e de Aprendizagem, de modo que se faz necessário estimular o desenvolvimento destas nos ambientes escolares, independentemente do histórico educacional dos pais.

Uma limitação desse estudo é não ter condição de verificar se esses estudantes que declararam não saber qual o nível de estudos de seus pais e de suas mães tinham essas duas figuras presentes em suas vidas. A ausência de uma dessas pessoas, devido a inúmeros aspectos como morte, não conhecimento dos pais, distância entre os entes familiares, dentre outros, pode ter desencadeado essas respostas.

Estudos adicionais precisam ser desenvolvidos para conhecer melhor a realidade do ensino médio brasileiro. É dado que grande parte dos alunos de 
escola pública não tem o privilégio de muita diversidade de experiências prévias culturais e educacionais (Brenner, Dayrell \& Carrano, 2005) que favoreçam a construção de crenças de Autoeficácia Acadêmica robustas. É importante que novos estudos sejam feitos considerando diferentes aspectos para ampliar a compreensão da relação aqui destacada, bem como de outras relações que ajudem a entender o papel das famílias na construção das crenças de autoeficácia acadêmica. Kim e Park (2006) comentaram que em todos os níveis educacionais as percepções de autoeficácia acadêmica dos estudantes recebem contribuições do suporte social advindo da mãe e do pai. Sugerem-se pesquisas que visem investigar as relações entre pais e filhos considerando-se as atividades escolares e acadêmicas, bem como dos processos de decisão de carreira. Isso parece ser necessário, principalmente na adolescência, período em que os estudantes, cursando o ensino médio, também se deparam com a busca e preparo para a vida profissional e conseqüente desenvolvimento de carreira.

\section{REFERÊNCIAS}

Almeida, L. (2002). Facilitar a aprendizagem: ajudar os alunos a aprender a pensar. Psicologia Escolar e Educacional, 6(2),155-165.

Azzi, R. G., Guerreiro-Casanova, D., Dantas, M.A., \& Maciel, A.C. (2011) Academic Self-efficacy and Learning and Study Strategies: Brazilian students' perceptions. In: 15th Biennal of the international study association on teachers and teaching, Braga.

Azzi, R. G., \& Polydoro, S. A. J. (2010). O papel da autoeficácia e autorregulação no processo motivacional. In: E Boruchovitch, J.A Bzuneck \& S.E.R.Guimarães (Orgs.), Motivação para aprender: Aplicações no contexto educativo. (pp.126-144). Rio de Janeiro: Ed. Vozes.

Bandura, A. (1993). Perceived self-efficacy in cognitive development and functiong. Educational Psychologist, 28(2), 117-148.

Bandura, A. (1995). Exercise of personal and collective efficacy in changing societies. In: A. Bandura (Ed). Self-efficacy in changing societies (pp.1-45). Cambridge: Cambridge University Press.

Bandura, A. (1997). Self-efficacy, the exercise of control. New York: Freeman and Company. 
Bandura, A. (2006). Adolescent development from na agentic perspective. In: Pajares, F. \& Urdan, T. Self-efficay beliefs of adolescents (pp.143).Connecticut: Information Age Publishing.

Bartalo, L. (2006) Mensuração de Estratégias de Estudo e Aprendizagem de Alunos Universitários: Learning And Study Strategies Inventory (Lassi) Adaptação e Validação para o Brasil. Tese de Doutorado, Unesp, Marília, São Paulo.

Brenner, A.K., Dayrell, J. \& Carrano, P. (2005). Culturas do lazer e do tempo livre dos jovens brasileiros. In: Abramo, H.W. \& Branco, P. P.M.(Eds.), Retratos da Juventude brasileira: Análises de uma pesquisa nacional (pp.175-214). São Paulo: Ed. Perseu Abramo.

Brown, S.D. \& Lent, R.W. (2006). Preparing adolescents to make carrer decisions: a social cognitive perspective. In: Pajares, F. \& Urdan, T. (Ed), Self-efficacy beliefs of adolescents (pp.201 - 223). Information age publishing: Greenwich.

Figueira, A.P.C. (1994). Inventário de Estratégias de Estudo e de Aprendizagem - Learning and Study Strategies Inventory - (LASSI) Estudos de Adaptação. Psychologica, 12, 79-114.

Grenne, B. A., Miller, R. B., Crowson, H. M., Duke, B. L. \& Akey, K. L. (2004) Predicting high school students' cognitive engagement and achievement: contributions of classroom perceptions and motivation. Contemporary Educational Psychology, 29, 462-482.

Kim, U \& Park, Y.S. (2006) Factors influencing academic achievement in relational cultures. In: Pajares, F. \& Urdan, T. (Ed), Self-efficacy beliefs of adolescents (pp.267- 285). Information Age Publishing: Greenwich.

Lopes, A. R. (2010). Projetos vocacionais, crenças de autoeficácia e expectativas parentais em estudantes do 70 ano de escolaridade em situação de sucesso/insucesso escolar. Dissertação de Mestrado. Universidade de Lisboa, Portugal.

Ogliari, E. \& Rodrigues, E. (2011). País tem de alfabetizar 3,5 milhões de adultos para cumpri meta da ONU. In: Jornal O Estado de São Paulo. p. A23. Domingo, 10 de julho de 2011.

Polydoro, S.A.J. \& Guerreiro-Casanova, D.C. (2010). Escala de Autoeficácia na Formação Superior: construção e estudo de validação. Avaliação Psicológica, 9(2), 267-278. 
Schunk, D.H. \& Meece, J. L. (2006). Self-efficacy development in adolescences. In: F. Pajares, T. Urdan (Eds.), Self-efficacy beliefs of adolescents. (pp. 7196). IAP (Information Age Publishing). USA.

Schunk, D. H., Printich, P. R. \& Meece, J.L. (2008). Sociocultural Influence. In: D. H., Schunk, P. R. Printich \& J.L.Meece (Eds.), Motivation in education: Theory, research and applications. (pp.281-289). Columbus, Ohio: Person Mervill Prentice Hall.

Rosário, P., Mourão, R., Núñez, J.C., González-Pienda, J.A., Solano, P., \& Valle, A. (2007). Eficacia de un programa instruccional para la mejora de procesos y estrategias de aprendizaje en la enseñanza superior. Psicothema, 19(3), 353-358.

Rosário, P., Mourão, R., Soares, S., Chaleta, E., Gracio, L., Simões, F., Núñez \& J.C., Gonzalez-Pienda, J.A. (2005). Trabalho de casa, tarefas escolares, autorregulação e envolvimento parental. Psicologia em Estudo, Maringá, $10(3), 343-351$.

Valle, A., Cabanach, R., Gonzalez-Pienda, Núñez, Rodriguez \& Rosário, P. (2009). Perfiles motivacionales em estudiantes de secundaria: análisis diferencial em estrategias cognitivas, estratégias de autorregulacion y rendimiento acadêmico. Revista mexicana de psicologia, 26(1)113-124.

Valle, A., Cabanach, R., Rodríguez, S., Gerpe, M., Piñeiro, I. \& Rosário, P. (2008). Las Estrategias de Aprendizaje y Estudio: Bases Teóricas para la Intervención. Revista Multiárea, Modelos, Métodos y Estrategias de Aprendizaje, 3, 263-286.

Weinstein, C. E. \& Palmer, D.R. (1990). LASSI: Learning and study strategies inventory. Clearwater, FL: H\&H Publishing Co.

Zambom, M. P, Azzi, R. G., Polydoro, S. A. J., De Rose, T. M. \& GuerreiroCasanova, D. C. (2011, Junho). Relations entre perceptions de la structure de la classe, auto-efficacité, buts d'accomplissement, stratégies d'apprentissage et performance dans un groupe de brésiliens. Congrès 2011 de I'Association Francophone Internationale de Recherche Scientifique en Education, UNESCO. Paris, França.

Zimmerman, B.J. (1995). Self-efficacy and educational development. In: A. Bandura (Ed). Self-efficacy in changing societies (pp.1-45). Cambridge: Cambridge University Press. 
Zimmerman, B. J. \& Cleary, T. J. (2006). Adolescent's development of personal agency. The role of self-efficacy beliefs and self-regulatory skill. In: F. Pajares \& Urdan, T. (pp. 45-69). Self-efficacy beliefs of adolescents. IAP (Information Age Publishing). USA.

Contato: danielaguerreiro@yahoo.com.br,_marildapsi@uol.com.br, betazzi@uol.com.br

Recebido em: 03/03/2011

Revisado em: 05/05/2011

Aceito em: 26/05/2011 